\title{
Environmental context-dependent recognition memory using a short-term memory task for input
}

\author{
STEVEN M. SMITH \\ Texas A\&M University, College Station, Texas
}

\begin{abstract}
Although many studies have demonstrated that recall is better when tested in the learning environmental context (EC) than in a new EC, almost all of the studies have failed to find ECdependent memory when recognition was used to measure memory. Evidence of EC-dependent recognition, however, was found in the three experiments of the present study, in which an incidental short-term memory task was used for input of learned material. The results suggest that type of processing during input is a predictor of context-dependent recognition; material studied for a long-term memory test is not susceptible to background context effects, whereas material merely maintained for a short-term memory test is more apt to lead to EC-dependent recognition.
\end{abstract}

Even when there is no obvious or intended relationship between learning material and the background learning context, it has commonly been observed that recall tested in the learning context is better than recall tested in a new context (e.g., Godden \& Baddeley, 1975; Smith, 1979; Smith, Glenberg, \& Bjork, 1978). Similar patterns have been noted for various background contexts: the environment (e.g., Smith et al., 1978), a drug state (e.g., Eich, 1980), or a mood state (e.g., Bartlett \& Santrock, 1979). Although effects of environmental and state changes on recall have often been large and robust, recognition memory has usually appeared to be unaffected by changes in background environment (Eich, 1985; Godden \& Baddeley, 1980; Jacoby, 1983; Smith et al., 1978), drug states (Eich, 1980; Spear, 1978), or mood states (Bartlett \& Santrock, 1979).

There is, of course, substantial literature on the effects of verbal or semantic context upon recognition memory (e.g., Light \& Carter-Sobell, 1970; Reder, Anderson, \& Bjork, 1974; Tulving \& Thomson, 1973), mostly under the rubric of encoding-specificity theory. The manipulation of context in those studies, however, is qualitatively different from manipulation of background environments or background states. The meaningful connections among items and their verbal contexts in encoding-specificity studies are absent in studies of incidental background context. The more obvious differences between verbal and environmental contexts include that (1) verbal contexts bias the meanings of list words directly, whereas environmental contexts do not; (2) verbal context studies gener-

\footnotetext{
This research was supported by NIMH Grant No. 1 R01 MH39977-01 awarded to Steven M. Smith. Experiments 1 and 2 were reported at the 1984 meeting of the Psychonomic Society in San Antonio, TX. Thanks for assistance in collecting the data for the present study are due to Steve Blankenship, Jimmy Knetsch, Roc LaRocque, Louise Morgan, Susan Schulz, James Truemper, and Mark Wagner. Requests for reprints should be addressed to Steven Smith, Psychology Department, Texas A\&M University, College Station, TX 77843.
}

ally provide a specific contextual element for each target item, whereas environmental context studies generally use a single presentation context; and (3) in verbal context studies, the attention of participants is usually explicitly or implicitly directed to the context, whereas in most environmental context studies, attention is not directed to the context.

The first distinction is a particularly critical one in explaining effects of verbal context on recognition. Concepts must be selected from semantic memory to represent items both at the time of learning and during recognition testing. A semantically related context, then, could bias such selections to be either the same or different at the times of learning and recognition testing (e.g., Anderson \& Bower, 1974; Kintsch, 1974), thus having a contextdependent effect upon recognition. Because selection of a meaning of a list word or a test word is not obviously influenced by the background environmental context, environmental context-dependent recognition based on this mechanism should not occur.

The meaning selection process, however, is not the only process purportedly responsible for context-dependent recognition memory. In Kintsch's (1974) model, for example, list items and contextual stimuli are represented in the same episodic memory traces. On a recognition test, concepts are implicitly retrieved from semantic memory to represent test items, and the implicitly retrieved concepts are combined with elements of the test context to form a memory probe that is matched with previously encoded events. This matching process, a component process common to both recall and recognition, is enhanced when there is greater overlap between the learning context (an encoded component of event memories) and the text context (an encoded component of the memory probe). Reinstatement of the learning context, therefore, should enhance both recall and recognition by facilitating the matching process. The lack of environmental context-dependent memory (CDM) and state-dependent 
memory (SDM) with recognition tests appears to disconfirm such theories.

Failures to find effects of the incidental background context (environmental, state) on recognition memory prompted Baddeley (1982) to propose that a background environment will not affect recognition unless effort is given to processing list words and environmental features interactively. Context-dependent recognition has been noted in studies of semantic context (e.g., Thomson \& Tulving, 1970) and voice context (e.g., Geiselman \& Bjork, 1980); in both cases, contextual information was processed interactively with list words. Independent processing of list items and contextual elements, according to Baddeley, is what prevents EC-dependent recognition from occurring.

Eich (1980), Geiselman and Bjork (1980), Smith et al. (1978), and Spear (1978) have independently proposed similar descriptions of CDM/SDM as a function of the cues provided by the memory test. Smith et al. stated,

In free recall, where the experimenter provides few explicit retrieval cues, the subject must make use of varied sources of cues to provide access to the stimuli. Environmental context could be one such source of cues. In cued recall there is more specific retrieval information, and in recognition memory the subject is supplied with highly effective, explicit cues, thereby reducing his dependence on other cue sources. (p. 352)

Spear (1978) also pointed out that this trend in SDM or dissociative memory studies is the result of diminished importance of contextual cues when memory is tested with recognition, and Eich's (1980) account of the situation was similar to Spear's. Geiselman and Bjork (1980) referred to the effective explicit cues reinstated on a recognition test as "intraitem situational context," whereas they labeled the more incidental background context "extraitem situational context." According to Geiselman and Bjork, the effective intraitem cues reinstated on the typical recognition tasks minimize the effect of extraitem context cues.

Smith et al.'s (1978) explanation will be referred to as the cue-specificity hypothesis. "Specificity" refers to the number of encodings in which a cue is represented; the fewer items in which a cue is encoded, the more specific is the cue (Glenberg, 1979). A cue is more effective if it is more specific, since more specific cues are less overloaded (Watkins \& Watkins, 1975). In a typical listlearning study, a descriptive cue (containing information about a single item) is very specific, a structural cue (e.g., interitem associations, categories, subjective organizations) is less specific, and a background context cue (e.g., environmental, state) is most general of all, being included in encodings of all list items.

I propose that a general cue is more effective when more specific cue sources are restricted. On a typical recognition test, structural cues and descriptive cues are available. Descriptive cues result from encoding the stimuli provided on the recognition test. Structural cues may become available as follows: The words on a recognition test are typically presented in a single block of time. Relations among the words (formed at the time of study) may be reactivated by the occurrence of some of the studied words on the recognition test. Recognition of other studied words can then be facilitated by these activated relations. It may be possible to manipulate the amount of structural information provided by the recognition test, with the idea that recognition memory that is dependent on environmental context (EC) should be more likely to appear when use of structural cues is restricted.

An unexpected finding of EC-dependent recognition occurred in a study (Smith, 1985b) concerned with cognitive effects of immersion in a flotation tank. The learning procedure in that study consisted of 10 trials of a memory span task. On each trial, five words were spoken on a tape recording, followed by $10 \mathrm{sec}$ of an unfilled interval, at the end of which the five words were recalled. In that study, the memory span task used as an incidental learning procedure led to the expected context-dependent recall effect, and, surprisingly, there was a marginal context-dependent recognition effect as well.

Reasoning that the flotation tank might have induced such powerful state-dependent effects that even recognition memory was influenced, I hypothesized that the same procedure would not produce a context-dependent recognition effect when context variations involved room changes. Alternatively, the cue-specificity hypothesis states that the shallow processing involved in the memory span task should reduce encoding of specific structural and descriptive item cues, thus amplifying the relative contribution of general contextual associations on a recognition task. Therefore, EC-dependent recognition should occur with environmental changes if a memory span task is used for incidental learning. These hypotheses were tested in Experiment 1.

\section{EXPERIMENT 1}

The procedure used in Experiment 1 was as similar as possible to the procedure used in the earlier flotation tank study (Smith, 1985b), so that the results of the present room-change experiment could be more directly compared with results of the tank experiment. Therefore, subjects responded orally in the present study, as they did in the flotation tank study, and interpolated perceptual tasks occurred between input and recognition testing, as in the previous study.

\section{Method}

Subjects. Thirty-six volunteers from introductory psychology classes at Texas A\&M University fulfilled part of a course requirement with their participation. Nine participants were assigned randomly to each of the four experimental conditions.

Materials. One hundred common one-syllable four- and five-letter English nouns were selected from the Kucera and Francis (1967) word-frequency norms. Fifty of the words were randomly selected to be distractors on the recognition test. The other 50 words, used on the short-term memory (STM) task, were randomly assigned to 10 -word sets. Each 5-word set was spoken at a 1-sec rate on 
audiotape, followed by $10 \mathrm{sec}$ of silence and then the command "Recall." Ten seconds were allowed for recall. After the test, another 5-word set was spoken (again at a 1-sec rate), and so on until all 10 sets were presented. Stimuli were presented through speakers.

Subjects then took the Group Embedded Figures Test (GEFT; Oltman, Raskin, \& Witkin, 1971), which consists of 18 embeddedfigure problems in which subjects must trace over a simple form hidden within a complex pattern. High scores indicate field independence, an analytic ability.

The third task was the Restricted Classification Task (RCT), which consists of 36 stimuli (Ward, 1980). Each stimulus has three lines of dots that vary in spacing of dots and line length. Each item has two lines that are identical on only one dimension and two that are similar overall, but not equal on either dimension. Subjects must choose "the two that go together best" for each three-line item. Choices are classified as dimensional responses (analytic), similarity responses (holistic), or haphazard responses.

The two-alternative forced-choice recognition test consisted of 50 pairs of words spoken on audiotape at 5-sec intervals. Subjects were asked to say aloud the word from each pair that was on the STM test. Targets and distractors were randomly paired. For each pair, the order of target and distractor was randomly determined.

Design and procedure. Each subject participated individually in Experiment 1. Subjects began in either the cubicle or the classroom with the STM task. After each of the 10 five-word sets was heard on a tape recording played through speakers, there were $10 \mathrm{sec}$ of silence, followed by the command "Recall." Upon command, subjects spoke aloud the five words from a trial and prepared for the next trial. Responses were tape recorded.

After the STM task, all subjects moved to the other room and took the GEFT and RCT. These two tasks together took about $25 \mathrm{~min}$. After the GEFT and RCT were given, subjects left the second room and returned either to their first room (where the STM task occurred) or to their second room (where the GEFT and RCT occurred).

In the final test room, subjects were given first a free recall test, followed by a recognition test. Both tests tested memory for words previously presented on the STM task. On the free recall test, subjects were given $4 \mathrm{~min}$ to recall as many words as possible in any order. Subjects' spoken responses were tape recorded. On the recognition test, the pairs were heard on a recording, and subjects spoke aloud the word from each pair that they believed to be the "old" word (i.e., from the STM task).

Environmental contexts. One room was a bare $6 \times 6 \mathrm{ft}$ cubicle with a single table and two chairs. The other room used in Experiment 1 was a faculty office in the Texas A\&M University psychology department. It was heavily cluttered with two desks, file cabinets, bookshelves, photos on the wall, and a rug. There was one window.

\section{Results}

Two separate $2 \times 2$ (test context $\times$ input context) ANOVAs were computed, one using total number of words recalled, and another using number of recognition errors as the dependent measure. The test context (i.e., where subjects were tested) consisted of two levels: same context (SC) and different context (DC). The two input contexts (i.e., where incidental learning occurred) were the cubicle and the office.

The effect of test context in the analysis of recall scores was significant $[F(1,32)=7.09, p<.05, M S e=$ 19.52]. The SC group recalled an average of 3.77 more words than did the DC group, an improvement equal to
Table 1

Mean Number of Items Recalled and Recognition Errors in Experiment 1 as a Function of Test Context

\begin{tabular}{lcc}
\hline & \multicolumn{2}{c}{ Test Context } \\
\cline { 2 - 3 } & Same Context & Different Context \\
\hline Number Recalled & 9.94 & 6.17 \\
Recognition Errors & 7.50 & 11.28 \\
\hline
\end{tabular}

Note-There were 50 items for each measure.

$61 \%$ of the mean number recalled in the DC condition (Table 1). There was no effect of input context $[F(1,32)$ $=.97, p=.33, M S e=19.52]$, nor was there a test context $\times$ input context interaction $[F(1,32)=.83, p=.37$, $M S \mathrm{e}=19.52$ ]

There was also an effect of test context found in the ANOVA using number of recognition errors $[F(1,32)=$ $7.20, p<.05, M S \mathrm{e}=19.16]$. DC subjects committed $50 \%$ more errors than did SC subjects (Table 1). There was no effect of input context $[F(1,32)=.65, p=.42$, $M S e=19.16]$, nor was there an input context $\times$ test context interaction $[F(1,32)=1.07, p=.31, M S e=19.16]$.

To examine the importance of field dependence/independence in context-dependent recognition, subjects were sorted according to their GEFT scores into two groups: field independent ( 13 points and above) or field dependent (below 13 points). Two $2 \times 2 \times 2$ (test context $\times$ input context $\times$ field dependence) ANOVAs were computed, one for recall and one for recognition. No main effects or interactions with field dependence were found in either analysis.

\section{Discussion}

Environmental context-dependent recognition was found in Experiment 1 using an incidental STM learning task for a single list of words presented in a single room. There have been no previous significant findings of ECdependent recognition memory that have used a singlelist design, such as the design used in Experiment 1.

A number of characteristics of the design used to find EC-dependent recognition could have contributed to the noted effect. The present experiment rules out, however, the possibility that the effect could somehow have been caused by use of the flotation tank in a previous study (Smith, 1985b); in the present study, the ECs used were an office and a laboratory cubicle. Another possible contributor to the EC-dependent recognition effect could have been the interpolated tasks (GEFT and RCT) given prior to the memory tests in the DC test room. This procedure could have (1) caused the DC subjects to approach the final memory tests with the wrong mental set, expecting not a memory test, but a perceptual test, or (2) caused greater retroactive interference for DC subjects, if that material could have interfered with memory of list words.

Another potential cause of EC-dependent recognition observed in Experiment 1 was the previous free recall test, which found an advantage of SC over DC recall. The previous recall test could have given an advantage to the 
SC group on the subsequent recognition test by priming more words in the SC condition. An unpublished study I performed at the University of Oklahoma, however, used an intentional learning task, and tested recall before recognition in either SC or DC conditions. That study found a robust effect of test context for free recall $[F(1,56)=$ $11.57, p<.01$ ], with SC subjects recalling $22 \%$ more than DC subjects, yet for recognition, performance $\left(d^{\prime}\right)$ was nearly identical for the two groups $[F(1,56)<1.00]$. Even so, the potential contamination caused by the prior free recall test was eliminated in Experiments 2 and 3 by using only a recognition test without a previous recall test.

Alternatively, it is proposed that the critical condition for observing EC-dependent recognition in Experiment 1 was the use of the STM task for learning. This hypothesis was further examined in Experiments 2 and 3.

\section{EXPERIMENT 2}

Experiment 2 tested the hypothesis that interpolated tasks given in the DC test room were responsible for the finding of EC-dependent recognition in Experiment 1. This hypothetical cause of the effect can be broken down into two parts: (1) presence (vs. absence) of interpolated tasks, and (2) occurrence of interpolated tasks in the DC test room (vs. elsewhere). In Experiment 2 one third of the conditions (Group A) received interpolated tasks in the DC test room, as in Experiment 1 and in Smith's (1985b) study, one third had the tasks in a room different from both the input and test ECs (Group B), and one third had no interpolated tasks between input and testing (Group C). The test context for each of these three groups was either same (SC) or different context (DC).

Experiment 2 differed from Experiment 1 in other ways. In Experiment 1, subjects were alone with the experimenter, whereas in Experiment 2, subjects participated in groups of 4 to 6 persons at a time. Because of the group sessions used in Experiment 2, oral responding was replaced with written responding. Furthermore, in Experiment 1 presentation modes for input and test stimuli were the same (aural), whereas presentation modes for input and test stimuli in Experiment 2 were different: input presentation was aural, and test stimuli were presented visually.

\section{Method}

Subjects. One hundred sixteen volunteers from introductory psychology classes at Texas A\&M University fulfilled part of a course requirement with their participation. Separate sign-up sheets for each of the 12 experimental groups were posted, allowing students to sign up for any session of the experiment. (The numbers of subjects in each group are shown in Table 3.)

Materials. The STM and GEFT tests used in Experiment 2 were the same as those used in Experiment 1.

A visual search task was used for an interpolated task instead of the RCT used in Experiment 1. The visual search task required subjects to circle target letters embedded in columns of distractor letters. Ten seconds were given for each of 10 columns of letters.

The two-alternative forced-choice recognition test consisted of 50 pairs of words printed on a 10 -page test booklet. There were five pairs printed per page. Each pair contained one word from the STM task and a common one-syllable distractor word (see Experiment 1).

Design and procedure. Subjects participated in groups ranging from 4 to 6 students at a time. Beginning either in a cubicle or a classroom, subjects took the same STM test described in Experiment 1 , the only difference being that subjects in Experiment 2 wrote down their responses on each trial rather than speaking them aloud.

After the STM task, subjects in Group A went to the DC test room (for those beginning in the cubicle, the DC test room was the classroom; for those beginning in the classroom, the DC test room was the cubicle) for interpolated tasks (GEFT and visual search), which took about 20 min. Those in Group B were given the interpolated tasks in a different room, a small seminar room. Those in Group $\mathrm{C}$ were given no interpolated tasks; they were asked to wait quietly in the hall for $3 \mathrm{~min}$ before they were taken to their recognition test EC (see Table 2).

The room in which recognition testing occurred was either the same context (SC) as that in which input (the STM task) occurred, or it was a different context (DC). Subjects were given $4 \mathrm{~min}$ to complete the 50 -item recognition test booklet. They were instructed to circle the one item from each pair that they believed was on the earlier STM test.

Environmental contexts. One room used for input and testing was the same barren cubicle described in Experiment 1. The other input and testing room was a classroom with windows, a blackboard, and 25 desks. A small windowless seminar room with seating at tables for about 12 students was used for the interpolated activity in Group B.

\section{Results}

A $2 \times 2 \times 3$ (test context $\times$ input context $\times$ interpolated activity context) ANOVA was computed for number of errors on the recognition test. There was a significant effect of test context $[F(1,104)=4.36, p<.05, M S \mathrm{e}=$ 11.84]. The DC subjects committed $28 \%$ (1.21) more errors than did the SC subjects, over all groups (Table 3). The effect was the most pronounced in Group $\mathrm{C}$, the group with no interpolated tasks between input and testing; in that condition, DC subjects made $73 \%$ (2.63) more recognition errors than did the SC subjects. Smaller effects in the same direction were noted in the groups that performed interpolated tasks between input and testing (A and B); in those groups, DC subjects made $12 \%(.59)$

Table 2

Location of Treatment Conditions in Experiment 2

\begin{tabular}{clllc}
\hline Group & Learning EC & Interpolated EC & Test EC & Condition \\
\hline A & Cubicle & Classroom & Cubicle & SC \\
& Classroom & Cubicle & Classroom & SC \\
& Cubicle & Classroom & Classroom & DC \\
& Classroom & Cubicle & Cubicle & DC \\
& B & Seminar Room & Cubicle & SC \\
& Cubicle & Seminar Room & Classroom & SC \\
& Cubicle & Seminar Room & Classroom & DC \\
& Classroom & Seminar Room & Cubicle & DC \\
& Cubicle & (none) & Cubicle & SC \\
C & Classroom & (none) & Classroom & SC \\
& Cubicle & (none) & Classroom & DC \\
& Classroom & (none) & Cubicle & DC \\
\hline
\end{tabular}

Note-DC $=$ different context; $S C=$ same context. 
Table 3

Mean Number of Recognition Errors in Experiment 2 as a Function of Learning Context, Test Context, and Interpolated Activity

\begin{tabular}{llllr}
\hline & & \multicolumn{2}{c}{ Test Context } & \\
\cline { 3 - 4 } & & $\begin{array}{c}\text { Same } \\
\text { Cearning Context }\end{array}$ & \multicolumn{1}{c}{ Different } & \\
\hline Group A & Context & Context & DC-SC \\
& Cubsroom & $4.00(9)$ & $4.56(9)$ & .56 \\
& Both Rooms & $5.55(11)$ & $7.33(9)$ & .78 \\
Group B & Classroom & $3.20(10)$ & $2.45(11)$ & -.75 \\
& Cubicle & $4.44(9)$ & $6.60(10)$ & 2.16 \\
& Both Rooms & $3.79(19)$ & $4.43(21)$ & .64 \\
Group C & Classroom & $3.70(10)$ & $6.18(11)$ & 2.48 \\
& Cubicle & $3.40(9)$ & $6.25(8)$ & 2.81 \\
& Both Rooms & $3.58(19)$ & $6.21(19)$ & 2.63 \\
All Subjects & Both Rooms & $4.27(58)$ & $5.48(58)$ & 1.21 \\
\hline
\end{tabular}

Note-There were 50 items. Numbers of subjects in each condition are shown in parentheses. $\mathrm{DC}=$ different context; $\mathrm{SC}=$ same context.

more errors than did SC subjects. The test context $x$ interpolated activity context interaction was not significant, however $[F(2,104)=1.02, p>.10, M S \mathrm{e}=11.84]$.

There was an effect of input context $[F(1,104)=7.47$, $p<.01, M S e=11.84]$. Subjects who began in the classroom recognized more than those who began in the cubicle. The ANOVA did not find any other main effects or interactions that approached significance.

\section{Discussion}

The results of Experiment 2 replicate and extend the finding of EC-dependent recognition in Experiment 1. The overall level of performance was better in Experiment 2 than in Experiment 1, possibly because of the written, rather than oral, responding in Experiment 2. The magnitude of the EC-dependent recognition effect, however, was about the same in both experiments.

Experiment 2 was partly motivated by the hypothesis that interpolated tasks given in the DC test room caused the finding of EC-dependent recognition in Experiment 1 and in Smith's (1985b) study. This hypothesis was clearly disproven by the results of Experiment 2, in which Group C, the group with no interpolated tasks, showed the greatest EC-dependent recognition. Neither interpolated tasks nor oral responding was necessary for observing EC-dependent recognition following a shallow processing task. The procedure of Experiment 2 omitted both oral responding and interpolated tasks, and ECdependent recognition was still observed.

The retention interval, or time between the learning and test sessions, is another factor whose importance is not clear. Although the findings of EC-dependent recognition in Smith's (1985b) study and in Experiment 1 of the present study were based upon 20-min retention intervals, the group showing the largest EC effect in Experiment 2 was Group $C$, which had only a 3 -min retention interval. Whatever the importance of the retention interval, the results of Experiment 2 show that EC-dependent recognition does not depend upon a 20-min retention interval.

\section{EXPERIMENT 3}

The finding of EC-dependent recognition in Experiments 1 and 2 appears to have been unaffected by interpolated tasks and response modes. What, then, caused ECdependent recognition to be observed in Experiments 1 and 2 , and was missing from procedures used in other studies where EC-dependent recognition was not observed (e.g., Godden \& Baddeley, 1980; Smith et al., 1978)? The type of processing devoted to learning may be the critical factor in this case. As compared with shallow processing, deeper processing of information results in the encoding of better memory cues. The cuing effect of the background EC was predicted to be greater for shallowly processed material than for material studied for a long-term memory test.

Experiment 3 tested the idea that type of input processing may affect the finding of EC-dependent recognition. Approximately one half of the subjects in Experiment 3 received short-term memory (STM) instructions, using a shallow processing procedure identical to that used for Group C of Experiment 2. The only difference between subjects given STM instructions and those given long-term memory (LTM) instructions in Experiment 3 was that those in the LTM condition were told, before hearing the list words, that they șhould try to memorize the list words to prepare for a later memory test; STM subjects were not told about the final test.

\section{Method}

The materials, design, procedure, and environments in Experiment 3 were identical to those used in Experiment 2 for the $C$ condition, with one exception. Thirty-nine subjects were given STM instructions (i.e., they were given the same instructions as subjects in Experiments 1 and 2), and 38 were given LTM instructions (i.e., they were given STM instructions, plus they were told that words would be tested again on a later test) prior to hearing the words. Thus, the design was a $2 \times 2 \times 2$ (test context $\times$ input context $\times$ learning instructions).

Subjects. The 77 subjects in Experiment 3 were volunteers from introductory psychology classes at Texas A\&M University who fulfilled part of a course requirement by participating in the experiment. Subjects were randomly assigned to experimental conditions.

\section{Results}

A $2 \times 2 \times 2$ (test context $\times$ input context $\times$ learning instruction) ANOVA was computed, using number of recognition errors as the dependent measure.

There was a marginally significant effect of test context $[F(1,69)=2.90, p=.09, M S e=12.05]$. DC subjects made $26 \%$ (1.33) more recognition errors than did SC subjects.

The EC-dependent recognition effect was due entirely to subjects given STM instructions, as in Experiments 1 and 2 . The test context $\times$ learning instruction interaction was significant $[F(1,69)=3.88, p=.05, \mathrm{MSe}=12.05]$. No EC effect whatsoever was seen in the LTM condition (Table 4), whereas an EC effect about the size of that found in Experiments 1 and 2 was found in the STM con- 
dition. In the STM condition, DC subjects committed an average of 59\% (2.76) more errors than did SC subjects. The EC effect in the STM condition, however, was not quite significant according to a Newman-Keuls test (critical difference $=3.40, \alpha=.05$ ).

Although there was no main effect of input context $[F(1,69)=.25]$, there were two significant interactions that involved the input context factor. The input context $\times$ learning instruction interaction was significant $[F(1,69)$ $=12.76, p<.01, M S e=12.05$ ]; under STM learning instructions, those learning in the classroom recognized more words than those learning in the cubicle, whereas with LTM instructions, the pattern was reversed. The test context $\times$ input context $\times$ learning instruction interaction was also significant $[F(1,69)=13.68, p<.01$, $M S \mathrm{e}=12.05]$. This three-way interaction indicates that the test context $\times$ learning instruction interaction (ECdependent recognition for STM instructions, not for LTM instructions) was caused by subjects who learned in the cubicle, not by those learning in the classroom (Table 4).

Those given LTM instructions made fewer recognition errors (5.63) than those given STM instructions (6.15). This learning instruction effect, however, was not significant $[F(1,69)=.34, p=.56, M S e=12.05]$.

The possibility that a floor effect for errors may have caused the test context $\times$ learning instruction interaction was examined by dividing each of the four groups at their medians into high-error subjects and low-error subjects. A $2 \times 2 \times 2$ (test context $\times$ learning instruction $\times$ subject type) ANOVA was conducted, using recognition errors as the dependent measure. There were significant effects only for test context $[F(1,69)=4.26, p<.05$, $M S e=6.63]$, for subject type $[F(1,69)=103.79$, $p<.001, M S e=6.63$ ], and for the test context $\times$ learning instruction interaction $[F(1,69)=5.34, p<.05$, $M S e=6.63]$. Subject type did not interact with any variables. Although Table 5 suggests that high-error subjects showed somewhat stronger EC-dependent recognition effects in the STM condition than did low-error subjects (suggesting a floor effect), the test context $\times$ learning instruction $\times$ subject type interaction was not significant $[F(1,69)=.87, p>.05, M S e=6.63]$. A floor effect may also be suggested by the finding that the three groups scoring nearer the floor (Groups STM-SC, LTM-SC, and LTM-DC) had smaller standard deviations $(3.03,3.33$,

Table 4

Mean Number of Recognition Errors in Experiment 3 as a Function of Test Context, Learning Instructions, and Input Context

\begin{tabular}{|c|c|c|c|c|}
\hline \multirow{2}{*}{$\begin{array}{c}\text { Input } \\
\text { Context }\end{array}$} & \multicolumn{2}{|c|}{$\begin{array}{l}\text { STM Learning } \\
\text { Instructions }\end{array}$} & \multicolumn{2}{|c|}{$\begin{array}{l}\text { LTM Learning } \\
\text { Instructions }\end{array}$} \\
\hline & SC & $\mathrm{DC}$ & $\mathrm{SC}$ & $\mathrm{DC}$ \\
\hline Cubicle & $3.89(9)$ & $10.80(10)$ & $5.09(11)$ & $3.00(9)$ \\
\hline Classroom & $5.44(9)$ & $4.36(11)$ & $6.44(9)$ & $8.11(9)$ \\
\hline Both Rooms & $4.67(18)$ & $7.43(21)$ & $5.70(20)$ & $5.50(18)$ \\
\hline
\end{tabular}

Note-There were 50 items. Numbers in parentheses indicate numbers of subjects in each condition. $\mathrm{SC}=$ same test context; $\mathrm{DC}=$ different test context.
Table 5

Mean Numbers of Recognition Errors in Experiment 3 as a Function of Learning Instructions, Test Context, and Subject Type

\begin{tabular}{lccccc}
\hline & \multicolumn{2}{c}{$\begin{array}{l}\text { STM Learning } \\
\text { Instructions }\end{array}$} & & \multicolumn{2}{c}{$\begin{array}{c}\text { LTM Learning } \\
\text { Instructions }\end{array}$} \\
\cline { 2 - 3 } \cline { 5 - 7 } Subject Type & SC & DC & & SC & DC \\
\hline High-Error Subjects* & 7.22 & 11.18 & & 8.30 & 8.44 \\
Low-Error Subjects* & 2.11 & 3.30 & & 3.10 & 2.67 \\
\hline
\end{tabular}

Note-There were 50 items. *High-error subjects scored above the median of their experimental treatment group, and low-error subjects scored below the median. $\mathrm{SC}=$ same test context; $\mathrm{DC}=$ different test context.

and 3.63, respectively) than the higher error group (STM$\mathrm{DC}, S D=5.46$ ).

\section{Discussion}

The results of Experiment 3 help resolve the discrepancy between previous failures to find EC-dependent recognition memory and the present findings of ECdependent recognition. The significant test context $\times$ learning instruction interaction indicates that although intentional learning (LTM) instructions do not lead to EC-dependent recognition, as in previous studies of the phenomenon, shallow incidental learning (STM) instructions may result in an EC-dependent recognition effect. The results are anomalous, however, due to the test context $x$ input context $\times$ learning instruction interaction. EC-dependent recognition in the STM condition was found only for those who learned in the cubicle, not for those who learned in the classroom. The EC-dependent recognition effects in Experiments 1 and 2 did not interact with input context; ECdependent effects were found regardless of where learning occurred. The failure to find the effect in one counterbalancing condition of Experiment 3 is not easily explained. Still, the overall trend showing EC-dependent recognition in the STM condition of Experiment 3 is consistent with the results of Experiments 1 and 2 .

The possibility that a floor effect for errors may have caused the test context $\times$ learning instruction interaction to reach significance seems unlikely in light of the analysis of high- and low-error subjects. Whether subjects were high in errors or near the floor in errors, the same pattern appeared; those given STM instructions showed ECdependent recognition, and those given LTM instructions did not.

Although the effect of learning instruction was not significant, the LTM instructions led to fewer errors than did STM instructions. The reason for the lack of an effect of learning instruction in the SC condition is not clear.

\section{GENERAL DISCUSSION}

Three experiments that used a shallow processing task for input of learning materials found clear evidence of EC-dependent recognition memory. Previous studies that used intentional learning tasks (Godden \& Baddeley, 1980; Smith et al., 1978), as well as the LTM instructed group in Experiment 3 of the present study, have failed 
to find EC-dependent recognition memory. The generality of the present findings is not known; the same set of materials and similar procedures were used in all three experiments. On the other hand, the findings were not limited to paradigms that used interpolated tasks or peculiar modes of responding.

The cue-specificity hypothesis predicts that EC cues will be more effective when more specific cue sources are restricted. Intentional learning is likely to provide highly specific memory cues in the form of structural cues (interitem associations) and elaborated descriptive information, whereas incidental shallow processing is less likely to result in the encoding of such specific cues that differentiate an encoded list item from other information in memory. The present finding of EC-dependent recognition following a shallow processing task, then, is consistent with the cue-specificity hypothesis.

A procedure intended to restrict use of structural cues on a recognition test was used by Smith (1985a). Multiple lists (two or more) were presented for intentional (LTM) learning, each in a different room, and recognition testing over all lists occurred in one of the learning rooms. On the recognition test, words from all input lists were randomly ordered, requiring subjects' memory judgments to alternate from one list to another, preventing adequate reinstatement of any one set of structural cues. The two experiments reported found small, but significant context-dependent recognition effects. A very similar twolist procedure (each list was learned in a different mood state) was used by Gage and Safer (1985) to study affectdependent recognition. They found that when testing occurred in the left visual field (connected with the hemisphere that purportedly encodes affect), recognition of facial expressions was affected by subject's induced mood. Their finding, like Smith's (1985a), was based upon a two-list randomized test procedure. Again, as in Smith's (1985a) study, this test procedure, which forced subjects' attention to alternate between different lists, could have minimized the reinstatement of structural cues on the recognition test, even though the LTM instructions may have induced subjects to encode structural cues.

Another type of explanation of context effects, to be referred to as the interactive hypothesis, states that the degree to which contextual information is processed interactively with target information will determine the degree of context-dependent recognition observed. This interactive hypothesis is described in essentially the same way by a number of investigators (e.g., Baddeley, 1982; Geiselman \& Bjork, 1980; Godden \& Baddeley, 1980). In Baddeley's (1982) explanation of EC-dependent memory, recognition was explained as being unaffected by an incidental background EC because such a context is encoded independently of the item (word) encodings. Only items processed interactively with their contexts should lead to context-dependent recognition, according to this explanation. Geiselman and Bjork (1980) had a similar explanation of context-dependent recognition, using the ideas of intraitem and extraitem context rather than interactive and independent processing of context; intraitem context refers to characteristics of the target item as presented, and supposedly influences recognition, whereas extraitem context refers to incidental aspects of the environment, and supposedly does not affect recognition memory.

The interactive hypothesis predicts that if intraitem context is processed and encoded interactively with list items, then context-dependent recognition should result, and if extraitem context is processed and encoded independently of list words, then context-dependent recognition should not result. Although there is evidence in support of this theory, there is also some disconfirming evidence. Eich (1985) found no evidence of EC-dependent recognition memory when subjects learned by forming images of list words interacting with objects in the learning environment, even though the same procedure produced a robust EC-dependent recall effect. Interactive intraitem context did not affect recognition. Furthermore, the present experiments, as well as those reported by Smith (1985a), show that independent extraitem EC can influence recognition.

The results of the present experiments suggest that there may be no need to distinguish between intraitem and extraitem context to explain findings and lack of findings of context-dependent recognition. Rather, the important factor to be considered may be the type of processing used during input, as suggested by the cue-specificity hypothesis. In previous studies (Godden \& Baddeley, 1980; Smith et al., 1978), intentional learning procedures were used, as in the LTM condition of Experiment 3, and none has shown an EC-dependent recognition effect. With a shallow processing task, however, as in the present experiments, EC-dependent recognition was found. The results of Geiselman and Bjork (1980) also exemplify such a pattern; voice context was unimportant for recognition following secondary rehearsal, but there was a voice contextdependent recognition effect following primary rehearsal.

Geiselman and Bjork (1980) suggested that secondary rehearsal improves retrieval of items probed by the recognition test, whereas primary rehearsal does not aid retrieval. According to this explanation, items difficult to recognize on a test are given a retrieval check. If such an item were learned with primary rehearsal, the retrieval check should be facilitated by contextual reinstatement. If such an item were learned with secondary rehearsal, the retrieval check should be facilitated by deep processing, regardless of the presence or absence of relatively weaker EC cues.

Retrieval is not the only process that may be affected by depth of processing and test context on a recognition test. A matching process (e.g., Kintsch, 1974), which compares test items with items stored in memory, could also be affected. If encoded items provide a good amount of information that can be used in such a matching process, the relatively little additional information provided by contextual cues may be functionally negligible. Items given maintenance rehearsal, however, may 
provide very little information with which to carry out this matching process, making contextual cues relatively important for matching and, therefore, for recognition.

Regardless of which theoretical account of contextdependent recognition is correct, the present results, combined with the findings of previous studies, provide three conclusions: (1) contrary to previous findings and assertions, EC-dependent recognition memory does occur; (2) EC-dependent recognition memory is more likely to be observed if learning material is merely maintained for a STM test, rather than studied in preparation for a LTM test; and (3) environmental (extraitem) context appears to function as a memory cue in the same way that other nonmeaningful forms of context (e.g., speaker's voice) cue memory.

\section{REFERENCES}

ANDERSON, J. R., \& Bower, G. H. (1974). A propositional theory of recognition memory. Memory \& Cognition, 2, 406-412.

Baddeley, A. D. (1982). Domains of recollection. Psychological Review, 89, 708-729.

Bartlett, J. C., Santrock, J. W. (1979). Affect-dependent episodic memory in young children. Child Development, 5, 513-518.

Erch, J. E. (1980). The cue-dependent nature of state-dependent retrieval. Memory \& Cognition, 8, 157-173.

EICH, J. E. (1985). Context, memory, and integrated item/context imagery. Journal of Experimental Psychology: Learning, Memory, \& Cognition, 11, 764-770.

GAGE, D. F., \& SAFER, M. A. (1985). Hemisphere differences in the mood state-dependent effect for recognition of emotional faces. Joumal of Experimental Psychology: Learning, Memory, \& Cognition, 11, $752-763$.

Geiselman, E. G., \& BJoRk, R. A. (1980). Primary versus secondary rehearsal in imagined voices: Differential effects on recognition. Cognitive Psychology, 12, 188-205.

GlenberG, A. M. (1979). Component-levels theory of the effects of spacing of repetitions on recall and recognition. Memory \& Cognition, 7, 95-112.

GodDEN, D. R., B BADDELeY, A. D. (1975). Context-dependent memory in two natural environments: On land and underwater. British Journal of Psychology, 66, 325-331.
Godden, D. R., \& Baddeley, A. D. (1980). When does context influence recognition memory? British Journal of Psychology, 71, 99-104.

JACOBY, L. L. (1983). Perceptual enhancement: Persistent effects of an experience. Joumal of Experimental Psychology: Human Leaming, Memory, \& Cognition, 9, 21-38.

KINTSCH, W. (1974). The representation of meaning in memory. Hillsdale, NJ: Erlbaum.

KuCERA, H., \& FranCIS, W. N. (1967). Computational analysis of present-day American English. Providence, RI: Brown University Press.

Light, L. L., \& CARTER-Sobell, L. (1970). Effects of changed semantic context on recognition memory. Journal of Verbal Learning \& Verbal Behavior, 9, 1-11.

Oltman, P. K., Raskin, E., \& Witkin, H. A. (1971). Group Embedded Figures Test. Palo Alto, CA: Consulting Psychologists Press.

Reder, L. M., ANderson, J. R., \& BJoRK, R. A. (1974). A semantic interpretation of encoding specificity. Journal of Experimental Psychology, 14, 632-649.

SMITH, S. M. (1979). Remembering in and out of context. Journal of Experimental Psychology: Human Learning \& Memory, 5, 460-471.

SMITH, S. M. (1985a). Environmental context and recognition memory reconsidered. Bulletin of the Psychonomic Society, 23, 173-176.

SMITH, S. M. (1985b, May). Memory and cognition in a flotation tank. Paper presented at the meeting of the Southwestern Psychological Association, Austin, TX.

SMITH, S. M., Glenberg, A. M., \& BJork, R. A. (1978). Environmental context and human memory. Memory \& Cognition, 6, 342-353.

SPEAR, N. E. (1978). The processing of memories: Forgetting and retention. Hilisdale, NJ: Erlbaum.

Thomson, D. M., \& Tulving, E. (1970). Associative encoding and retrieval: Weak and strong cues. Journal of Experimental Psychology, 86, 255-262.

Tulving, E., Thomson, D. M. (1973). Encoding specificity and retrieval processes in episodic memory. Psychological Review, 80, 352-373.

WARD, T. B. (1980). Separable and integral responding by children and adults to the dimensions of length and density. Child Development, 51, 676-684.

WATKINS, O. C., \& WATKINS, M. J. (1975). Buildup of proactive inhibition as a cue-overload effect. Journal of Experimental Psychology: Human Learning \& Memory, 1, 442-453.

(Manuscript received June 25, 1985; revision accepted for publication February 3, 1986.) 